\title{
Mutations in MC1R Gene Determine Black Coat Color Phenotype in Chinese Sheep
}

\author{
Guang-Li Yang, ${ }^{1}$ Dong-Li Fu, ${ }^{2}$ Xia Lang, ${ }^{3}$ Yu-Tao Wang, \\ Shu-Ru Cheng, ${ }^{2}$ Su-Li Fang, ${ }^{2}$ and Yu-Zhu Luo ${ }^{2}$ \\ ${ }^{1}$ Department of Life Sciences, Shangqiu Normal University, Shangqiu 476000, China \\ ${ }^{2}$ Gansu Province Key Laboratory of Herbivorous Animal Biotechnology, Gansu Agricultural University, Lanzhou 730070, China \\ ${ }^{3}$ Lanzhou Institute of Animal and Veterinary Pharmaceutics Sciences, Chinese Academy of Agricultural Sciences, \\ Lanzhou 730050, China
}

Correspondence should be addressed to Yu-Zhu Luo; luoyz@gsau.edu.cn

Received 29 June 2013; Accepted 26 July 2013

Academic Editors: N. L. Tang and A. Walley

Copyright (c) 2013 Guang-Li Yang et al. This is an open access article distributed under the Creative Commons Attribution License, which permits unrestricted use, distribution, and reproduction in any medium, provided the original work is properly cited.

\begin{abstract}
The melanocortin receptor 1 (MC1R) plays a central role in regulation of animal coat color formation. In this study, we sequenced the complete coding region and parts of the $5^{\prime}$ - and $3^{\prime}$-untranslated regions of the MC1R gene in Chinese sheep with completely white (Large-tailed Han sheep), black (Minxian Black-fur sheep), and brown coat colors (Kazakh Fat-Rumped sheep). The results showed five single nucleotide polymorphisms (SNPs): two non-synonymous mutations previously associated with coat color (c.218 T>A, p.73 Met>Lys. c.361 G>A, p.121 Asp>Asn) and three synonymous mutations (c.429 C> T, p.143 Tyr > Tyr; c.600 T>G, p.200 Leu >Leu. c.735 C> T, p.245 Ile>Ile). Meanwhile, all mutations were detected in Minxian Black-fur sheep. However, the two nonsynonymous mutation sites were not in all studied breeds (Large-tailed Han, Small-tailed Han, Gansu Alpine Merino, and China Merino breeds), all of which are in white coat. A single haplotype AATGT (haplotype3) was uniquely associated with black coat color in Minxian Black-fur breed $(P=9.72 E-72$, chi-square test). The first and second A alleles in this haplotype 3 represent location at 218 and 361 positions, respectively. Our results suggest that the mutations of $M C 1 R$ gene are associated with black coat color phenotype in Chinese sheep.
\end{abstract}

\section{Introduction}

Animal coloration is an ideal model for studying the genetic mechanisms that determine phenotype [1]. Coat color in domestic animals is one of the most strikingly variable and visible traits and has been widely used as a unique phenotype in the morphological selection for breed identification and attribution. In a large number of mammalian species, the coat color diversity is mainly determined by the relative amount of two basic melanins, eumelanin (black/brown), and pheomelanin (yellow/red), which are genetically controlled by the Extension (E) and Agouti (A) loci, respectively [2]. The Agouti locus encodes for the agouti signalling protein (ASIP) [3], a small paracrine signaling molecule that interacts with the product of the Extension locus. The $E$ locus encodes the melanocortin receptor 1 (MC1R), which is a seventransmembrane domains protein belonging to the G-protein coupled receptor present on the surface of the melanocyte membrane [4].

Functional mutations of the MC1R gene causing variation in coat colors have been described in domestic animals, such as cattle [5], pigs [6, 7], horses [8], goats [9], and sheep [10-13]. A notable example is the conserved role of the $M C 1 R$ in mammalian pigmentation [14]. Studies of $M C 1 R$ have provided valuable insights not only into the biology of pigmentation but also the evolution of domesticated animals $[15,16]$.

China has more than 40 native sheep breeds [17]. During the long-term selective breeding, it has resulted in diverse coat color phenotypes in Chinese indigenous breeds, including black, white, and brown pigment types. There are three ecosystem sheep groups (Kazak, Tibetan, and Mongolian) in China as well as other local populations or breeds (Minxian Black-fur, Small-tailed Han, Large-tailed Han, Tan, Gansu 


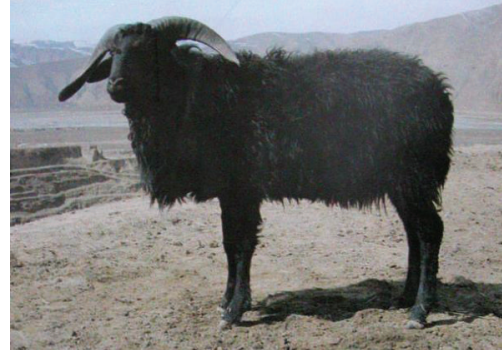

(a)

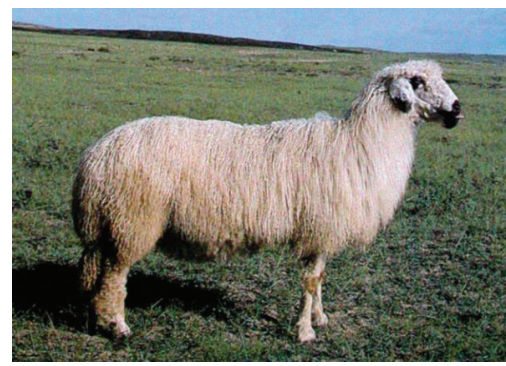

(d)

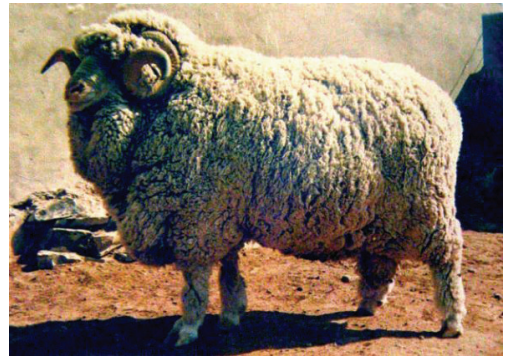

(g)

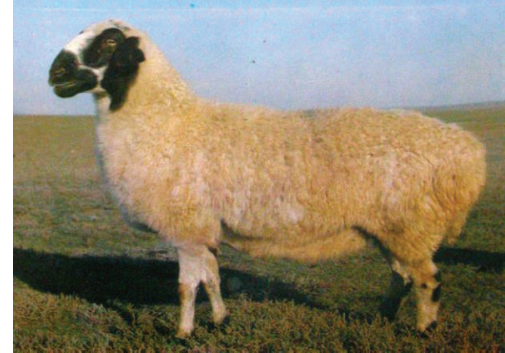

(b)

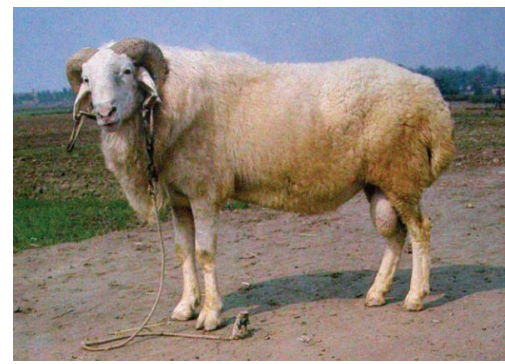

(e)

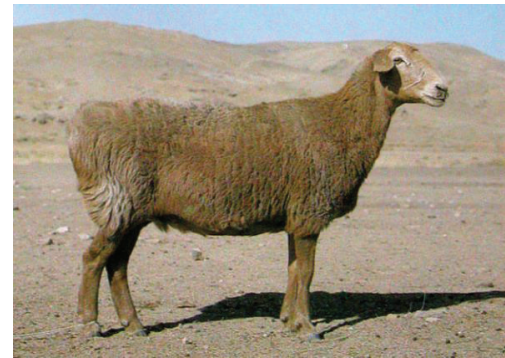

(h)

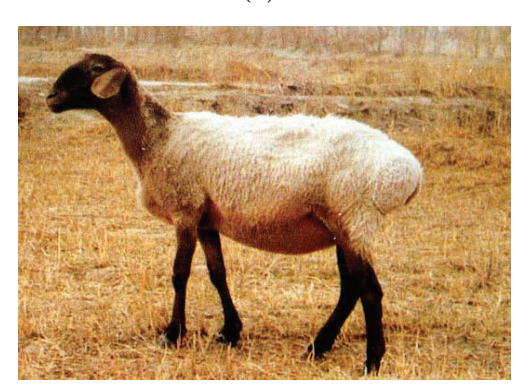

(j)

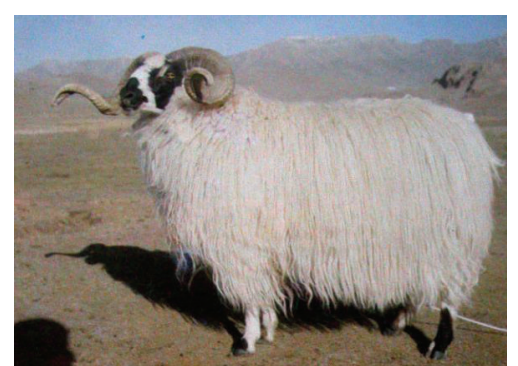

(c)

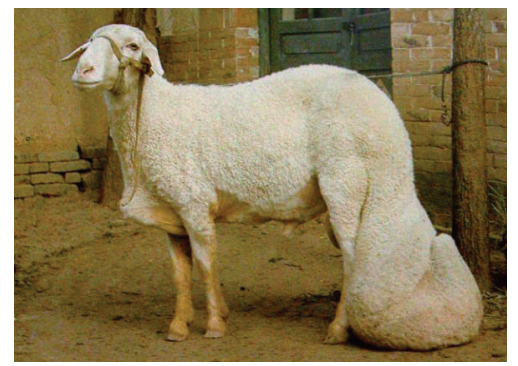

(f)

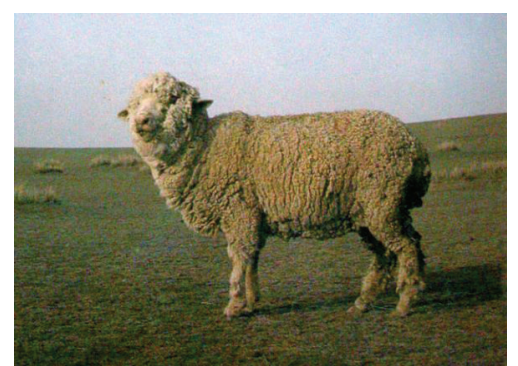

(i)
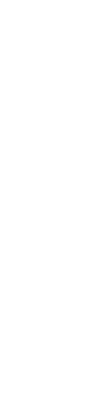

FIGURE 1: Illustration of sheep coat colors. (a) Minxian Black-fur sheep: black; (b) Mongolian sheep: white coat with black or brown face; (c) Tibetan sheep: white coat with black or brown face; (d) Tan sheep: white coat with black or brown face; (e) Small-tailed Han sheep: white; (f) Large-tailed Han: white; (g) China Merino: white; (h) Kazakh Fat-Rumped: Brown; (i) Gansu Alpine Merino: white; (j) Duolang sheep: white or gray coat with black or brown face.

Alpine Merino, China Merino, and Duolang) [17]. In most breeds, all animals share the same color pattern as breed character, such as Minxian Black-fur, Kazakh Fat-Rumped, and Mongolian sheep breeds (Ujimqin, Bayinbuluk, Wuranke, Sunite, and Hulun Buir) [17] (Figure 1). The Minxian Blackfur sheep is the predominant among Chinese indigenous breed that has uniform black coat [17].

In sheep, at least ASIP, MCIR, and TYRP1 genes have been implicated in coat color $[10-13,18-21]$. There are lots of papers describing the effect of $M C 1 R$ gene in coat color trait [10-13]. MC1R gene is located on chromosome 14 (OAR14) in sheep [11] and has three main alleles $\left(E^{+}, E^{D}\right.$, and $e$ ), which are defined by three mutations in the coding region and associated with variation in coat color [10-12]. However, so far there is no report regarding the $M C 1 R$ gene and the potential association of its mutations with coat colors in Chinese indigenous sheep. Therefore, we characterized the $M C 1 R$ gene by sequencing DNA pools comprising 30 sheep 
TABLE 1: Sample collection: breed name, sample size, coat color phenotype, and sampling location.

\begin{tabular}{lccc}
\hline Breed & Number & Coat color phenotype & Sampling location \\
\hline Minxian Black-fur & 46 & Black & Gansu, Min county \\
Tibetan & 42 & White, with black or brown face & Qinghai, Hainan county \\
Large-tailed Han & 48 & White & Henan, Jia county \\
Small-tailed Han & 34 & White & Shandong, Heze county \\
Mongolian & 51 & White, with black or brown face & Mongolian \\
Tan & 45 & White, with black or brown face & Gansu, Jintai county \\
Kazakh Fat-Rumped & 18 & Brown & Xinjiang, Akesu \\
Gansu Alpine Merino & 34 & White & Gansu, Sunan county \\
China Merino & 27 & White & Xinjiang, Yili state \\
Duolang & 28 & White or gray, with black or brown face & Xinjiang, Maigaiti county \\
\hline
\end{tabular}

individuals belonging to three native breeds with different coat colors: Minxian Black-fur sheep (Black), Large-tailed Han (White), and Kazakh Fat-Rumped (Brown) (Figure 1) and subsequent analysis of mutations in 10 different Chinese sheep breeds. The purpose of this study was to investigate the variability in $M C 1 R$ and their possible association with the coat color in Chinese sheep breeds.

\section{Material and Methods}

2.1. Animals. A total of 373 blood samples were collected from 10 Chinese sheep breeds representing a range of distinct coat colors (Figure 1). Breed name, sample size, coat color phenotype, and sampling location for each breed were shown in Table 1. Coat colors were determined by direct visual inspection. Genomic DNA was extracted from blood specimens by using the TIANamp blood DNA kit (Tianjin, Beijing, China).

2.2. SNPs Identification and Genotyping. SNPs were identified by sequencing amplicons of the whole coding domain sequences (CDS, $954 \mathrm{bp}$ ) and parts of the $5^{\prime}$ - and $3^{\prime}$ untranslated regions ( 35 and $125 \mathrm{bp}$, resp.) of $M C 1 R$ in both directions. Three DNA pools comprise thirty individuals with 10 individuals DNA $(100 \mathrm{ng} / \mu \mathrm{L}, 5 \mu \mathrm{L}$ for each individual) from each breed of Large-tailed Han sheep (White), Minxian Black-fur sheep (Black), and Kazakh Fat-Rumped sheep (Brown) and were used for identification mutation sites. Primers (MF: GAGAGCAAGCACCCTTTCCT, MR: GAGAGTCCTGTGATTCCCCT) for MC1R amplification and sequencing were designed with the program Primer 3 (http://fokker.wi.mit.edu/) based on the published coding region sequences in sheep (GenBank accession number: Y13965) and the complete sequences in bovine and goat which include $5^{\prime}$ - and $3^{\prime}$ - untranslated flanking regions (GenBank accession numbers: AF445641 and FM212940).

All amplifications were performed on Eppendorf Mastercycler (Hamburg, Germany). The reaction was performed in a total of $25 \mu \mathrm{L}$ containing $50 \mathrm{ng}$ DNA template (DNA pools), $100 \mu \mathrm{M}$ dNTPs, $10 \mathrm{pM}$ of MC1R specific primers (MF and $\mathrm{MR}$ ), and 2.5 U Taq polymerase (Bocai, Shanghai, China). After denaturation at $94^{\circ} \mathrm{C}$ for $3 \mathrm{~min}, 35$ amplification cycles were performed comprising a denaturation step at $94^{\circ} \mathrm{C}$ for
$30 \mathrm{~s}$ and an annealing step at $62^{\circ} \mathrm{C}$ for $30 \mathrm{~s}$, an extension at $72^{\circ} \mathrm{C}$ for $45 \mathrm{~s}$, followed by a last extension at $72^{\circ} \mathrm{C}$ for $10 \mathrm{~min}$. The PCR products were separated and visualized by electrophoresis on $1.5 \%$ agarose gels ethidium bromide staining. PCR products were purified with the QIAquick PCR Purification Kit (Qiagen, Hilden, Germany). Sequences were analyzed using DNAStar software (DNAStar Inc., Madison, WI, USA) to identify polymorphisms. Identified highly informative SNPs were (minor allele frequencies $>0.3$ ) chosen for genotyping by sequencing in a larger sample of animals belonging to the 10 breeds. PCR amplification and SNPs genotyping were performed as described above.

2.3. Data Analysis. Deviations from Hardy-Weinberg equilibrium (HWE) between SNPs were tested by POPGENE 3.1 [22]. Haplotypes of the SNPs within MC1R gene were determined using the PHASE program v. 2.1 [23]. The association analyses between haplotypes and coat colors were performed using crosstabs with fisher exact test implemented in the procedure descriptive statistics with the SPSS version 16.0 software (SPSS Inc. Chicago, IL, USA).

\section{Results}

3.1. SNPs Identification and Genotyping. By analysing and comparing the obtained sequence electropherograms from DNA pools of 30 sheep individuals. The results showed that five single nucleotide polymorphisms (SNPs), two nonsynonymous mutations previously associated with coat color (c.218 T>A, p.73 Met>Lys. c.361 G>A, p.121 Asp>Asn) and three synonymous mutations (c.429 C>T, p.143 Tyr>Tyr; c.600 T>G, p.200 Leu>Leu. c.735 C>T, p.245 Ile>Ile), were identified in the CDS of MC1R gene (Figure 2) (GenBank accession number: KF198511). These polymorphisms were reported by Våge et al. [10] and Fontanesi et al. [12]. However, we did not find recessive allele $e$ (c.199 C>T), which was reported by Fontanesi et al. [12].

These SNPs were further screened in a larger number of animals of 10 Chinese sheep breeds. Genotypes and allele frequencies were shown in Table 2. A chi-square test showed that 10 breeds were in Hardy-Weinberg equilibrium, while Kazakh Fat Rumped and Minxian Black-fur breed showed significant $(P<0.05)$ and very significant $(P<0.01)$ 


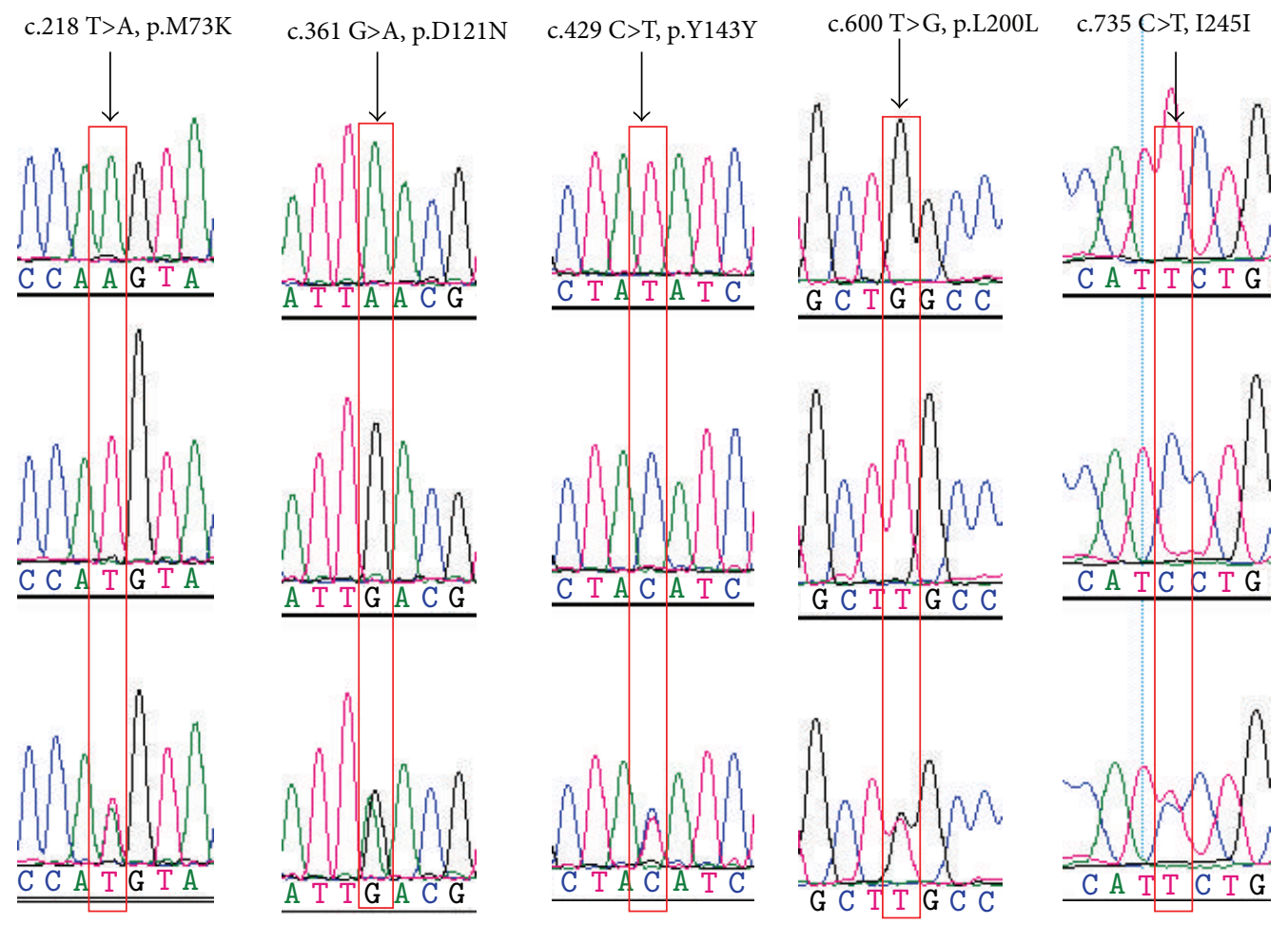

FIGURE 2: Identified SNPs and alignment of the MC1R protein regions around the deduced amino acid substitutions.

departures from Hardy-Weinberg equilibrium at MC1R c.218 $\mathrm{T}>\mathrm{A}$ and $M C 1 R$ c.361 G>A.

All mutation alleles (c.218A, c.361A, c.429T, c.600G, and c.735T) were detected in Minxian Black-fur sheep breed, and each mutation site has two genotypes. In particular, two nonsynonymous mutations (c.218 T>A, p.73 Met>Lys. c.361 $\mathrm{G}>\mathrm{A}, \mathrm{p} .121$ Asp $>$ Asn) determining the dominant black $\left(E^{D}\right)$ allele [10], were not at all identified in Large-tailed Han, Small-tailed Han, Gansu Alpine Merino, and China Merino, all of which are in white coat color. The Kazakh Fat-Rumped and Mongolian populations have three genotypes for each nonsynonymous mutation loci. But two nonsynonymous mutations frequencies were very low or rare in Kazakh FatRumped, Mongolian, and other three Chinese native sheep breeds (Tibetan, Tan, and Duolang). Three silent mutations in other sheep breeds, except for Minxian Black-fur and Kazakh Fat-Rumped breeds, have three genotypes. Interestingly, in this study, we found almost all mutation alleles in the Minxian Black-fur sheep breed at a rather high frequency $(0.6630$ and 0.8913 ). Three silent mutation alleles have also higher frequency (0.667) than two nonsynonymous mutation sites (0.333) in Kazakh Fat-Rumped breed.

3.2. Haplotype. Table 3 reports individual diplotype types and haplotype frequencies among the investigated breeds. Three haplotypes (haplotype1 [TGCTC], haplotype2 [TGTGT], and haplotype3 [AATGT]) and six individual diplotype types (haplotypel/haplotypel, haplotypel/haplotype2, haplotype 2/haplotype2, haplotype1/haplotype3, haplotype2/haplotype3, and haplotype3/haplotype3) were identified. The haplotype 3 of the all individual mutations was observed only in the Minxian Black-fur sheep breed. 17 Minxian Black-fur sheep were homozygous for haplotype3/haplotype3, 29 Minxian Black-fur sheep were heterozygous for haplotypel/haplotype3 (10), and for haplotype2/haplotype3 (19). The white coat color breeds (Largetailed Han, Small-tailed Han, Gansu Alpine Merino, and China Merino) were only found in three diplotype types (haplotype1/haplotype1, haplotypel/haplotype2, and haplotype2/haplotype2). The Mongolian has six diplotype types. Tan and Kazakh Fat-Rumped breeds have five diplotype types. Tibetan and Duolang have four similar diplotype types. Interestingly, we also observed that the haplotype 3 frequency was the highest in Minxian Black-fur sheep population (0.6848). The haplotype3 was absent in four Chinese sheep breeds with white coat (Large-tailed Han, Small-tailed Han, Gansu Alpine Merino, and China Merino) and was very low (0.0119-0.333) in other five Chinese indigenous breeds (Tibetan, Mongolian, Tan, Kazakh Fat-Rumped, and Duolang).

3.3. Association Analysis. Among a total of 373 Chinese sheep individuals, 46 were black coat color phenotype (Minxian Black-fur) and 18 were classified as brown (Kazakh FatRumped). The genotyping and haplotyping data (Tables 2 and 3) clearly indicated that polymorphisms in the $M C 1 R$ gene affect coat color in Minxian Black-fur. First of all, all animals with a mutation sites haplotype3 (46) showed uniform apparent black coat color, and almost all animals without a mutation sites haplotype3 (143) were completely white coat color (Large-tailed Han, Small-tailed Han, Gansu 


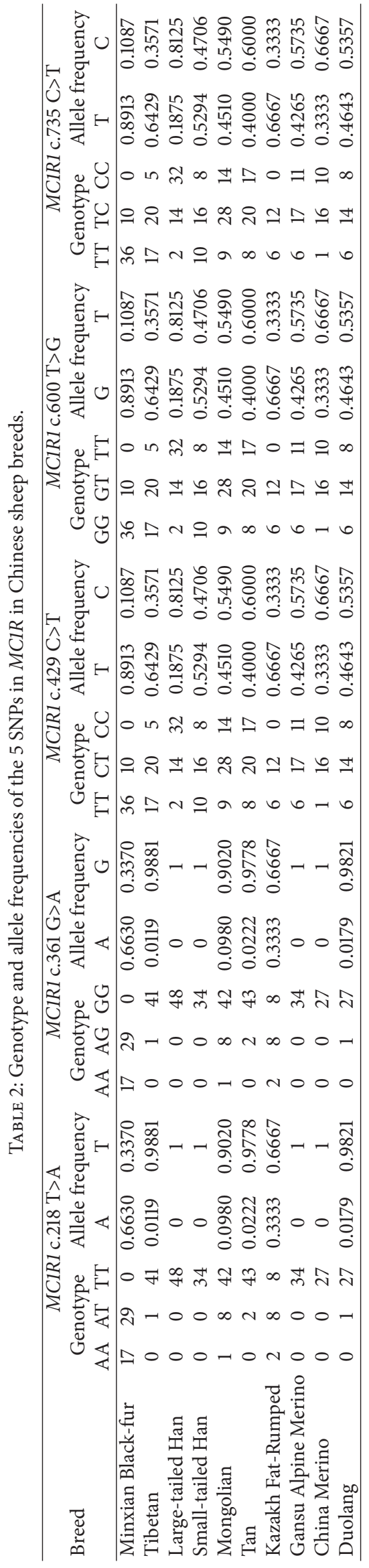


TABLE 3: Haplotype and haplotype frequencies at MC1R in 10 Chinese sheep breeds.

\begin{tabular}{|c|c|c|c|c|c|c|c|c|c|}
\hline \multirow{2}{*}{ Breed } & \multicolumn{6}{|c|}{ Haplotype } & \multicolumn{3}{|c|}{ Haplotype frequency } \\
\hline & $1 / 1$ & $1 / 2$ & $2 / 2$ & $1 / 3$ & $2 / 3$ & $3 / 3$ & Haplotype1 [TGCTC] & Haplotype2 [TGTGT] & Haplotype3 [AATGT] \\
\hline Minxian Black-fur & 0 & 0 & 0 & 10 & 19 & 17 & 0.1087 & 0.2065 & 0.6848 \\
\hline Tibetan & 5 & 18 & 18 & 0 & 1 & 0 & 0.3571 & 0.6310 & 0.0119 \\
\hline Large-tailed Han & 32 & 14 & 2 & 0 & 0 & 0 & 0.8125 & 0.1875 & 0.0000 \\
\hline Small-tailed Han & 8 & 16 & 10 & 0 & 0 & 0 & 0.4706 & 0.5294 & 0.0000 \\
\hline Mongolian & 14 & 22 & 6 & 6 & 2 & 1 & 0.5490 & 0.3529 & 0.0981 \\
\hline Tan & 17 & 19 & 7 & 1 & 1 & 0 & 0.6000 & 0.3778 & 0.2222 \\
\hline Kazakh Fat-Rumped & 0 & 7 & 1 & 5 & 3 & 2 & 0.3333 & 0.3333 & 0.3333 \\
\hline Gansu Alpine Merino & 11 & 18 & 5 & 0 & 0 & 0 & 0.5735 & 0.4265 & 0.0000 \\
\hline China Merino & 9 & 16 & 2 & 0 & 0 & 0 & 0.6667 & 0.3333 & 0.0000 \\
\hline Duolang & 8 & 14 & 5 & 0 & 1 & 0 & 0.5357 & 0.4464 & 0.0179 \\
\hline
\end{tabular}

Notes: Haplotypes are indicated following the SNP positions in the MClR gene: c.218 T>A, c.361 G>A, c.429 C>T, c.600 T>G, and c.735 C > T.

Alpine Merino, and China Merino). Secondly, the association analyses between haplotypes and coat colors are also showing that all the mutation alleles of haplotype 3 were highly significantly associated with Minxian Black-fur coat color $(P=9.72 E-72)$. But only a few animals did not follow the above rules: twenty-one of 184 (13/166 white coat color with black or brown patches in the head and 8/18 brown animals) carried out haplotype3. The alleles of haplotype3 have not been associated with white coat color with black or brown patches in the head (Tibetan, Mongolian, Tan, and Duolang) and brown coat color (Kazakh Fat-Rumped) animals.

\section{Discussion}

Classical genetic studies had proved two alleles $\left(E^{D}\right.$ and $\left.E^{+}\right)$ at the Extension locus affecting sheep coat color phenotypes $[2,24]$. Subsequently, Våge et al. [10] characterized two missense mutations (p.M73 K and p.D121N) determining the dominant black $\left(E^{D}\right)$ allele in the Norwegian Dala breed. The presence of two mutations was also observed in other sheep breeds: Corriedale, Damara, Black Merino, Black Castellana, and Karakul [11, 19]. The allele $E^{D}$ was directly involved in affecting sheep pigmentation at the molecular level and causes the dominant black coat color.

The recessive $e$ allele of the Extension locus has also been clearly documented in sheep. One SNP (c.199 C>T) caused a predicted amino acid substitution (p.R67C) in a highly conserved position of the first intracellular loop of the MC1R protein [12]. The same substitution causes recessive pheomelanism in other species $[7,25]$. Therefore, they propose that the p.67C allele represents the recessive $e$ allele at the sheep Extension series that was not completely recognized in sheep by classical genetic studies. This polymorphism was analysed in Italian sheep breeds or populations. Confirming the effect of this novel allele on coat color will lead to new perspectives.

Chinese sheep breeds have more variations on coat color among and, in some cases, within breeds. Therefore, five SNPs were also identified in Chinese sheep breeds in the MC1R gene by direct sequencing (Figure 2). The recessive allele $e$ (c.199 C>T), which has been linked to the control coat color in sheep, was not detected in the Chinese sheep. Two of five polymorphisms (c.218 T>A and c.361 G>A) were deduced as nonsynonymous substitutions causing a p.M73K and the p.D121N amino acid change, respectively. In the sheep, two amino acid (p.M73K and p.D121N) changes resided in the extracellular second transmembrane region (p.M73K) and in the third transmembrane domain (p.D121N) [10]. Both mutations in sheep have been associated with coat color variation. Additionally, both mutations could explain the dominant black coat color in sheep $[10,11,19]$.

Five SNPs were genotyped in 10 Chinese sheep breeds with different coat color phenotypes. All mutations were detected in Minxian Black-fur sheep breed, and nonsynonymous mutation sites were not at all identified in white coat coloration breeds (Table 2). This finding demonstrated that five mutations were completely associated with the black coat color in Minxian Black-fur sheep population. Meanwhile, three haplotypes (haplotype1, haplotype2, and haplotype3) were defined by the mutations SNPs in the MC1R gene. It was interesting that haplotype 3 was almost fixed in the Minxian Black-fur sheep breed (two missense mutations causing the $E^{D}$ allele were inserted in a haplotype3). Other four completely white sheep breeds had not carried the haplotype3 (Table 3). Furthermore, association analysis also indicated that the alleles of haplotype3 were significantly associated with the black coat color $(P=9.72 E-72$, Chisquare test). Therefore, the alleles of haplotype 3 might be a possible result that can interpret black coat color mechanisms in the Minxian Black-fur sheep breed that shaped the genetic pool of this sheep breed.

In Kazakh Fat-Rumped, two nonsynonymous mutation sites have three genotypes, and three silent mutations results were in accordance with Minxian Black-fur sheep breed (Table 2). Moreover, this breed has five diplotype types (Table 3). Thus, it is worthwhile to caution that the brown phenotype in Kazakh Fat-Rumped breed seems not to be caused by the identified MC1R mutations. However, Gratten et al. [20] report the identification of the TYRP1 gene and 
causal mutation underlying coat color variation in a freeliving population of Soay sheep. They identified a nonsynonymous substitution in exon IV that was perfectly associated with coat color. This polymorphism is predicted to cause the loss of a cysteine residue that is highly evolutionarily conserved and likely to be of functional significance. They eliminated the possibility that this association is due to the presence of strong linkage disequilibrium with an unknown regulatory mutation by demonstrating that there is no difference in relative TYRP1 expression between color morphs. Analysis of this putative causal mutation in a complex pedigree of more than 500 sheep revealed almost perfect cosegregation with coat color and very tight linkage between coat color and TYRP1.

In addition, according to the phenotype observed in Chinese-Tibetan having the same brown coat color [26], Ren et al. [27] performed a genome-wide association study (GWAS) on Tibetan and Kele pigs and found that brown colors in Chinese breeds are controlled by a single locus on pig chromosome 1 . Then, by using a haplotype-sharing analysis, they refined the critical region to a $1.5 \mathrm{Mb}$ interval that encompasses only one pigmentation gene: TYRP1. Lastly, mutation screens of sequence variants in the coding region of TYRP1 revealed a strong candidate causative mutation (c.1484-1489del). The protein-altering deletion showed complete association with the brown coloration across ChineseTibetan, Kele, and Dahe breeds by occurring exclusively in brown pigs and lacking in all nonbrown-coated pigs from 27 different breeds. The findings provide the compelling evidence that brown colors in Chinese indigenous pigs are caused by the same ancestral mutation in TYRP1. Moreover, Beraldi et al. [28] have shown an effect of dilution of pigmentation in Soay sheep that maps to chromosome 2, in a region where the candidate gene for brown coat color, TYRP1, is located. Therefore, we can rule out the possibility of $M C 1 R$ mutations determining the brown coat color phenotype. The brown coat color phenotype in Kazakh Fat-Rumped sheep may be caused by TYRP1 gene mutations that need to be further investigated.

Tibetan, Duolang, Tan, and Mongolian breeds usually include completely white coat animals together with black or brown patches in the head (around the eyes and/or in the ears or cheeks) (Figure 1). According to results from genotype and haplotype, the same substitution and haplotype (haplotype frequencies) were present in Tibetan, Duolang, Tan, and Mongolian breeds. However, there was no complete association between the presence of black or brown spots in the face and the presence of the AATGT alleles or haplotype3. Fontanesi et al. [9] reported missense and nonsense mutations in $M C 1 R$ gene of different goat breeds. According to the results obtained that $M C 1 R$ mutations may determine eumelanic and pheomelanic phenotypes, however, they are probably not the only factors. In particular, the surprising not complete association of the nonsense mutation (p.Q225X) with red coat colour raises a few hypotheses on the determination of pheomelanic phenotypes in goats that should be further investigated. Sponenberg et al. [24] showed that the wild allele at the Spotting locus allows full extension of pigmentation with no white spotting. The spotting of the recessive allele usually involves the distal legs and top of head before other areas and tends to result in reasonably recognizable patterns of spotting. Adalsteinsson [29] also suggested that the variation in the spotted $\left(S^{S}\right)$ effect can be explained by the action of modifiers, and white head spot occurs in animal heterozygous for white markings by incomplete dominance of the dominant allele $\left(\mathrm{S}^{+}\right)$for full pigmentation. Hence, these (Tibetan, Duolang, Tan, and Mongolian) breeds were probably due to incomplete fixation of different alleles at the spotting locus. The spotting locus or other loci with similar phenotypic effects might act through inhibition or disregulation of melanocyte migration from the neural crest at the embryonic level. This complicates the interpretation of the results as a complete characterization of the spotting locus in sheep is lacking. Therefore, when spots are present it could be possible to evaluate if different mutations are associated with the presence of eumelanic or pheomelanic colors.

Norris and Whan [18] characterized the sheep ASIP gene showing that a $190 \mathrm{~kb}$ tandem duplication encompassing this gene, the AHCY coding region (CDS), and the ITCH promoter region should be the cause of the white coat colour of dominant white and $\tan \left(\mathrm{A}^{\mathrm{Wt}}\right)$ Agouti sheep. In addition, a not yet characterized regulatory mutation as well as a deletion of $5 \mathrm{bp}$ in exon 2 and a missense mutation in exon 4 was identified as the causes of the black recessive nonagouti $\left(\mathrm{A}^{\mathrm{a}}\right)$ allele $[18,19,21]$. Analysis of the ASIP gene was also performed in the same Chinese sheep breeds by Fu et al. [30] (in press). The results showed that two deletion mutations and three SNPs were identified: a $9 \mathrm{bp}$ deletion (c.10-18del) and 5-bp deletion (c.100-105del), both of which were located in exon 2, and three SNPs (g.672 G>A, g.1580 G>A and g.1617 G>A) were located in intron 2. Two deletion mutations were presented in 10 Chinese sheep breeds. Moreover, only two sheep have the $\mathrm{D}_{5} \mathrm{D}_{5}$ genotype, one in Minxian Blackfur sheep and one in Duolang sheep, and no homozygosis $\mathrm{D}_{9} \mathrm{D}_{9}$ was found in all sheep that we detected. The genotype results suggested that these mutations are not associated or not completely associated with coat color in the investigated sheep breeds. The above results indicated that the variation in the protein coding region of ASIP did not explain the coat colour phenotypes variation of Chinese indigenous sheep breeds. These investigated results are also proved evidence that the black coat color phenotype in Chinese sheep was caused by the $M C 1 R$ gene mutations.

\section{Conclusion}

The present study results further confirm that the $M C 1 R$ gene is an important candidate gene because its mutations are associated with black color phenotype in Chinese indigenous sheep breed. In addition, we can rule out the mutations of $M C 1 R$ determining the brown coat color phenotype.

\section{Authors' Contribution}

Guang-Li Yang and Dong-Li Fu contributed equally to this work and should be considered as cofirst authors. 


\section{Acknowledgments}

This study was supported by He'nan Research Program of Foundation and Advanced Technology of China (102300410143, 132300410398) and Foundation of He'nan Educational Committee of China (12B230011).

\section{References}

[1] J. K. Hubbard, J. A. C. Uy, M. E. Hauber, H. E. Hoekstra, and R. J. Safran, "Vertebrate pigmentation: from underlying genes to adaptive function," Trends in Genetics, vol. 26, no. 5, pp. 231239,2010

[2] A. G. Searle, Comparative Genetics of Coat Colour in Mammals, Logos Press, London, UK, 1968.

[3] S. J. Bultman, E. J. Michaud, and R. P. Woychik, "Molecular characterization of the mouse agouti locus," Cell, vol. 71, no. 7, pp. 1195-1204, 1992.

[4] L. S. Robbins, J. H. Nadeau, K. R. Johnson et al., "Pigmentation phenotypes of variant extension locus alleles result from point mutations that alter MSH receptor function," Cell, vol. 72, no. 6, pp. 827-834, 1993.

[5] F. Rouzaud, J. Martin, P. F. Gallet et al., "A first genotyping assay of french cattle breeds based on a new allele of the extension gene encoding the melanocortin-1 receptor (MC1R)," Genetics Selection Evolution, vol. 32, no. 5, pp. 511-520, 2000.

[6] J. M. H. Kijas, M. Moller, G. Plastow, and L. Andersson, "A frameshift mutation in MC1R and a high frequency of somatic reversions cause black spotting in pigs," Genetics, vol. 158, no. 2, pp. 779-785, 2001.

[7] J. M. H. Kijas, R. Wales, A. Törnsten, P. Chardon, M. Moller, and L. Andersson, "Melanocortin receptor $1(M C 1 R)$ mutations and coat color in pigs," Genetics, vol. 150, no. 3, pp. 1177-1185, 1998.

[8] L. Marklund, M. J. Moller, K. Sandberg, and L. Andersson, "A missense mutation in the gene for melanocyte-stimulating hormone receptor $(M C 1 R)$ is associated with the chestnut coat color in horses," Mammalian Genome, vol. 7, no. 12, pp. 895-899, 1996.

[9] L. Fontanesi, F. Beretti, V. Riggio et al., "Missense and nonsense mutations in melanocortin 1 receptor (MC1R) gene of different goat breeds: association with red and black coat colour phenotypes but with unexpected evidences," BMC Genetics, vol. 10, Article ID 47, 2009.

[10] D. I. Våge, H. Klungland, L. Dongsi, and R. D. Cone, "Molecular and pharmacological characterization of dominant black coat color in sheep," Mammalian Genome, vol. 10, no. 1, pp. 39-43, 1999.

[11] D. I. Våge, M. R. Fleet, R. Ponz et al., "Mapping and characterization of the dominant black colour locus in sheep," Pigment Cell Research, vol. 16, no. 6, pp. 693-697, 2003.

[12] L. Fontanesi, F. Beretti, V. Riggio et al., "Sequence characterization of the melanocortin 1 receptor $(M C 1 R)$ gene in sheep with different coat colours and identification of the putative $e$ allele at the ovine Extension locus," Small Ruminant Research, vol. 91, no. 2-3, pp. 200-207, 2010.

[13] L. Fontanesi, S. Dall'Olio, F. Beretti, B. Portolano, and V. Russo, "Coat colours in the Massese sheep breed are associated with mutations in the agouti signalling protein (ASIP) and melanocortin 1 receptor (MC1R) genes," Animal, vol. 5, no. 1, pp. 8-17, 2011.

[14] L. Andersson, "Melanocortin receptor variants with phenotypic effects in horse, pig, and chicken," Annals of the New York Academy of Sciences, vol. 994, pp. 313-318, 2003.
[15] M. Y. Fang, G. Larson, H. S. Ribeiro, N. Li, and L. Andersson, "Contrasting mode of evolution at a coat color locus in wild and domestic pigs," PLoS Genetics, vol. 5, no. 1, Article ID e1000341, 2009.

[16] J. Li, H. Yang, J.-R. Li et al., "Artificial selection of the melanocortin receptor 1 gene in Chinese domestic pigs during domestication," Heredity, vol. 105, no. 3, pp. 274-281, 2010.

[17] P. Q. Zheng, The Editorial Section of Sheep and Goat Breeds in China. Sheep and Goat Breeds in China, Shanghai, Scientific and Technical Publishers, Shanghai, China, 1989.

[18] B. J. Norris and V. A. Whan, "A gene duplication affecting expression of the ovine ASIP gene is responsible for white and black sheep," Genome Research, vol. 18, no. 8, pp. 1282-1293, 2008.

[19] L. J. Royo, I. Álvarez, J. J. Arranz et al., "Differences in the expression of the ASIP gene are involved in the recessive black coat colour pattern in sheep: evidence from the rare Xalda sheep breed," Animal Genetics, vol. 39, no. 3, pp. 290-293, 2008.

[20] J. Gratten, D. Beraldi, B. V. Lowder et al., "Compelling evidence that a single nucleotide substitution in TYRP1 is responsible for coat-colour polymorphism in a free-living population of Soay sheep," Proceedings of the Royal Society B, vol. 274, no. 1610, pp. 619-626, 2007.

[21] J. Gratten, J. G. Pilkington, E. A. Brown, D. Beraldi, J. M. Pemberton, and J. Slate, "The genetic basis of recessive selfcolour pattern in a wild sheep population," Heredity, vol. 104, no. 2, pp. 206-214, 2010.

[22] F. C. Yeh, R. C. Yang, and T. Boyle, "Popgene version 1.31 Microsoft Widows-based freeware for population genetic analysis," Quick User Guide, 1999.

[23] M. Stephens, N. J. Smith, and P. Donnelly, "A new statistical method for haplotype reconstruction from population data," American Journal of Human Genetics, vol. 68, no. 4, pp. 978989, 2001.

[24] D. P. Sponenberg, C. H. S. Dolling, R. S. Lundie, A. L. Rae, C. Renieri, and J. J. Lauvergne, "Coat colour loci (category 1)," in Mendelian Inheritance in Sheep (MIS 96), J. J. Lauvergne, C. H. S. Dolling, and C. Renieri, Eds., pp. 13-57, University of Camerino, Camerino, Italy, 1998.

[25] H. Klungland, D. I. Vage, L. Gomez Raya, S. Adalsteinsson, and $\mathrm{S}$. Lien, "The role of melanocyte-stimulating hormone (MSH) receptor in bovine coat color determination," Mammalian Genome, vol. 6, no. 9, pp. 636-639, 1995.

[26] H. R. Mao, J. Ren, N. S. Ding, S. J. Xiao, and L. S. Huang, "Genetic variation within coat color genes of MC1R and ASIP in Chinese brownish red Tibetan pigs," Animal Science Journal, vol. 81, no. 6, pp. 630-634, 2010.

[27] J. Ren, H. R. Mao, Z. Y. Zhang, S. J. Xiao, N. S. Ding, and L. S. Huang, "A 6-bp deletion in the TYRP1 gene causes the brown colouration phenotype in Chinese indigenous pigs," Heredity, vol. 106, no. 5, pp. 862-868, 2011.

[28] D. Beraldi, A. F. McRae, J. Gratten, J. Slate, P. M. Visscher, and J. M. Pemberton, "Development of a linkage map and mapping of phenotypic polymorphisms in a free-living population of soay sheep (Ovis aries)," Genetics, vol. 173, no. 3, pp. 1521-1537, 2006.

[29] S. Adalsteinsson, "Color inheritance in Icelandic sheep and relation between colour fertility and fertilization," Journal of Agricultural Research, vol. 2, no. 1, pp. 3-135, 1970.

[30] D. L. Fu, G. L. Yang, X. Lang et al., "Research on ASIP gene mutations in Chinese sheep breeds," Journal of Gansu Agriculture University, 2014 (Chinese). 

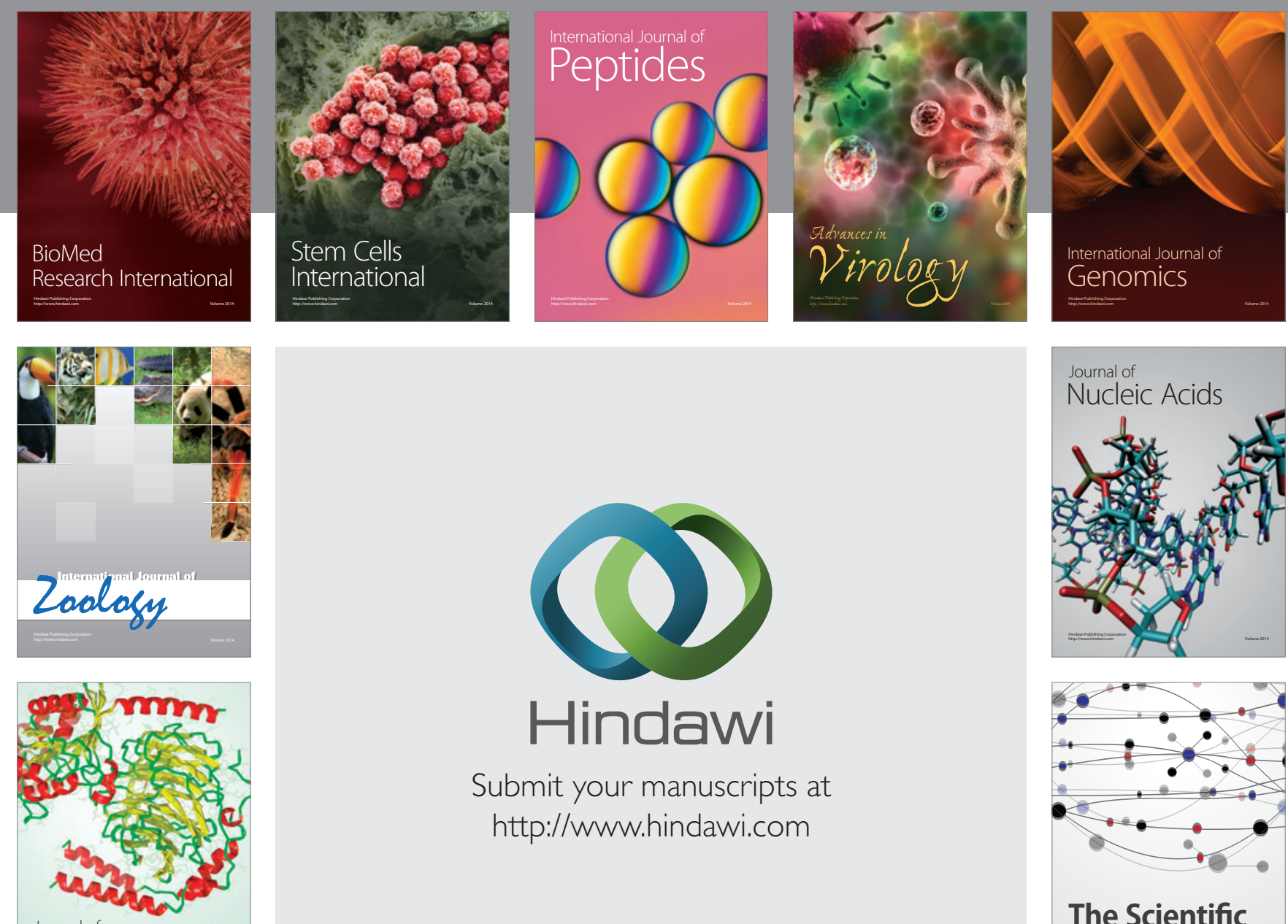

Submit your manuscripts at

http://www.hindawi.com

Journal of
Signal Transduction
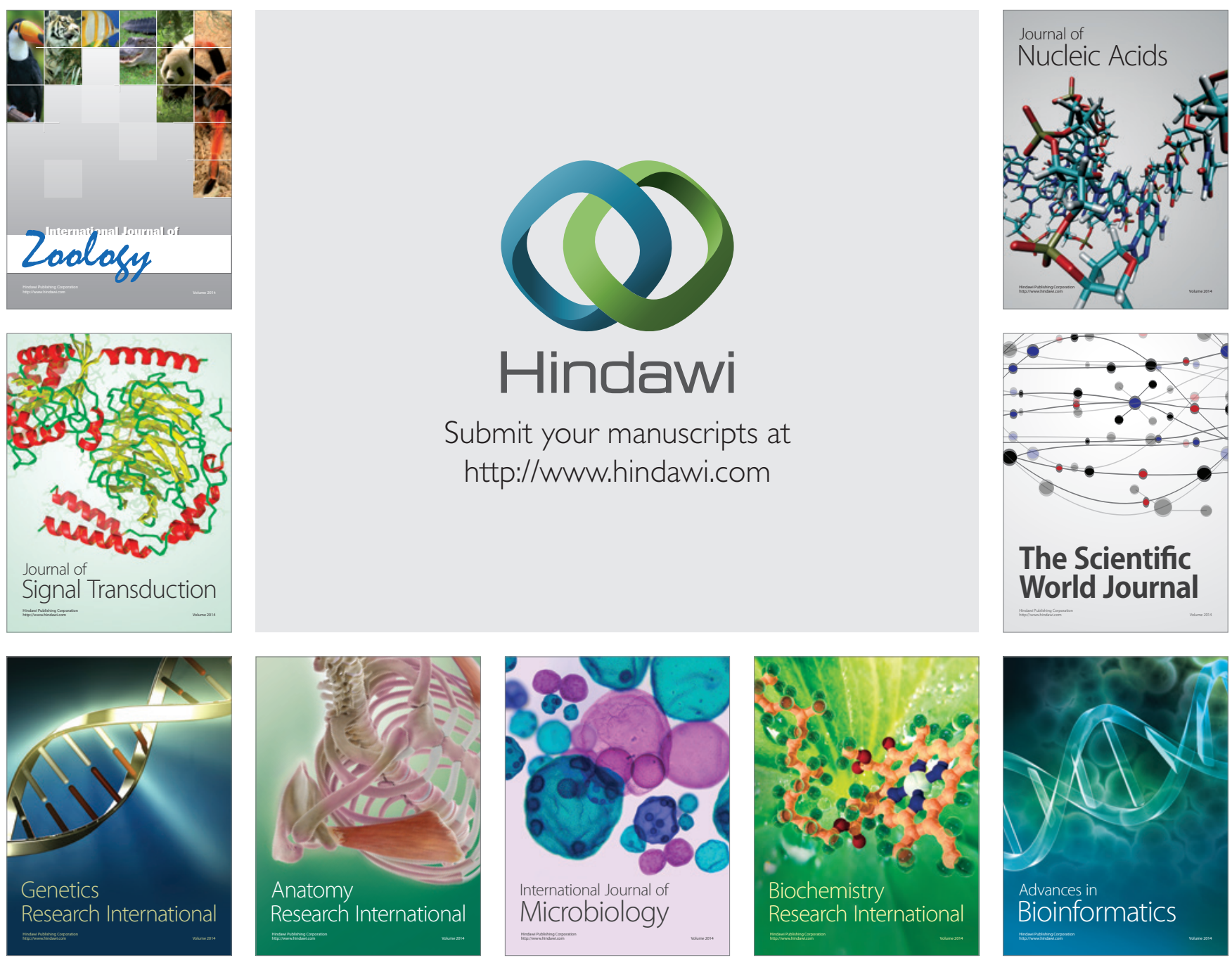

The Scientific World Journal
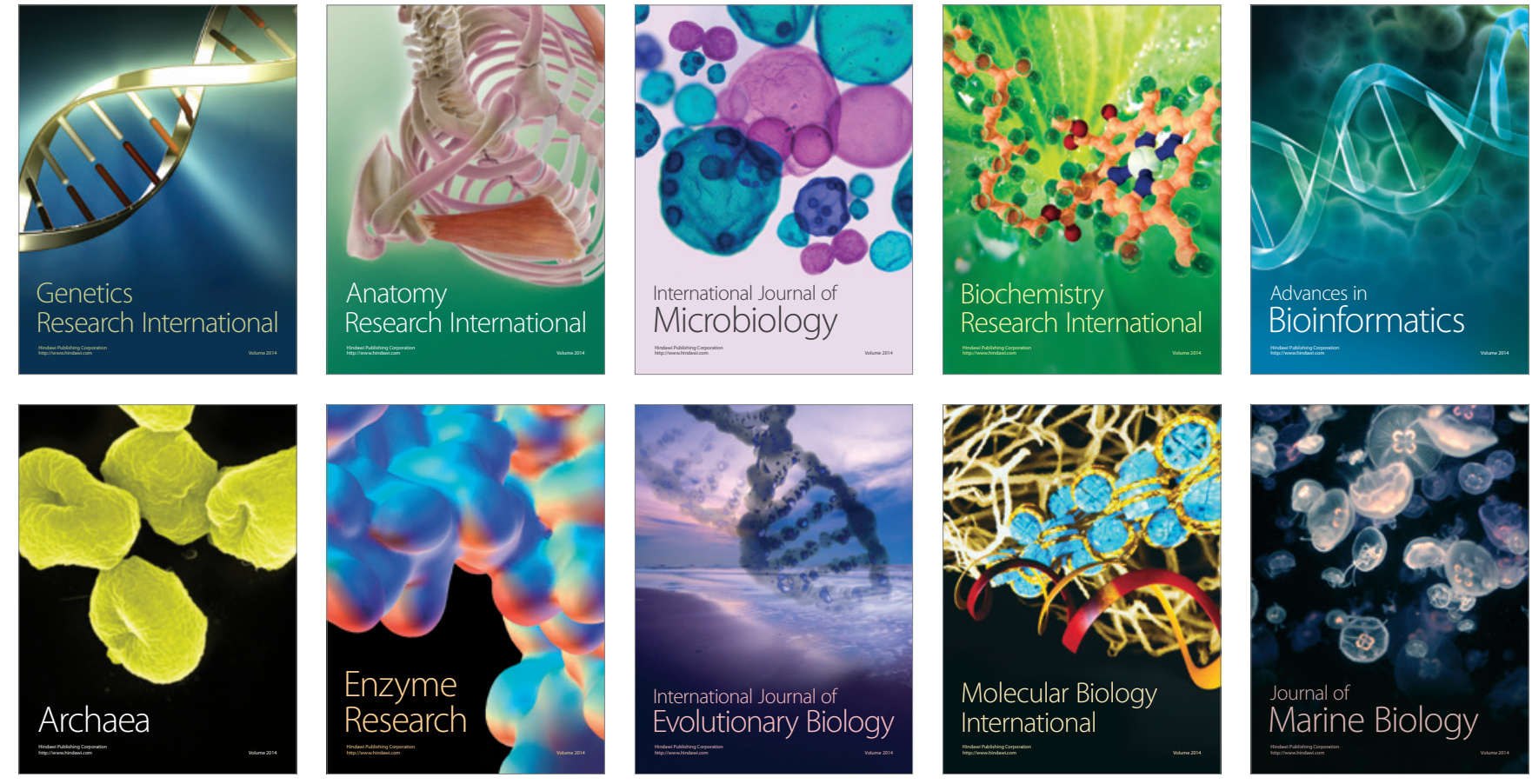